\title{
UTILIZACIÓN DE IMÁGENES DIGITALES PARA MEDICIÓN DEL DIÁMETRO DE FRUTOS DE MANDARINA (Citrus reticulata) EN CRECIMIENTO
}

\section{USING DIGITAL IMAGES FOR MEASUREMENT OF MANDARIN (Citrus reticulata) FRUITS DIAMETER DURING GROWTH}

\author{
Carlos Alberto Padrón-Pereira ${ }^{1}$ \\ ${ }^{1}$ Asociación RVCTA. Avenida Andrés Bello $N^{o}$ 101-79, Sector La Pastora, Municipio \\ Valencia, Estado Carabobo, C. P. 2001, República Bolivariana de Venezuela. \\ Emails: carlospadron1@gmail.com
}

\begin{abstract}
RESUMEN
$\mathrm{E}$ 1 propósito de este trabajo fue usar imágenes digitales para medir el diámetro ecuatorial de frutos de mandarina durante el crecimiento en el árbol para construir curvas de crecimiento. El experimento se realizó en una parcela ( $9 \mathrm{~m}$ x $5 \mathrm{~m}$ ), ubicada en Valencia (Venezuela) y cultivada con árboles frutales (Acerola, limón, mandarina, palmeras) creciendo naturalmente. Se seleccionaron dos frutos de la parte inferior de la copa (altura del suelo $2.5 \mathrm{~m}$ ) en crecimiento, de idéntico diámetro ecuatorial $(39 \mathrm{~mm})$ y en la cercanía se colocó una referencia fija de medida. Se utilizó una computadora portátil, una cámara digital calibrada y un software de diseño gráfico. El ensayo se llevó a cabo durante 105 días y cada 4 a 5 días se fotografiaron los frutos. Las imágenes captadas se descargaron en la computadora y procesaron con el software CorelDRAW ${ }^{\circledR}$ X3. La medición de los frutos en las imágenes se realizó con una regla graduada virtual creada con el software y con los datos obtenidos se elaboraron curvas de crecimiento. Los dos frutos se comportaron de similar manera durante el crecimiento, con coincidencias en las mediciones los días 58 y 84 (44.25 y $50.50 \mathrm{~mm}$, respectivamente), en este último día, aproximadamente se inició la fase 3, coincidiendo en ambos frutos. Los diámetros ecuatoriales alcanzados a los 105 días fueron 48.50 y $50.75 \mathrm{~mm}$ para el fruto 1 y 2 , respectivamente. Fueron factibles las mediciones mediante el procedimiento utilizado.
\end{abstract}

Palabras clave: Curvas de crecimiento de frutos, diámetro ecuatorial, mandarina, procesamiento digital de imágenes.

Recibido: 06-Febrero-2012. Recibido en forma corregida: 13-Febrero-2013. Aceptado: 09-Abril-2013.

Publicado como ARTÍCULO CIENTÍFICO en Ciencia y Tecnología 6(1): 1-9

Enero - Junio de 2013

ISSN 1390-4051 impreso; ISSN 1390-4043 electrónico

Abstract

$\mathrm{T}$ he purpose of this work was to use digital images for measuring the equatorial diameter of mandarin fruits during growth on the tree for the construction of growth curves. The experiment was carried out in a plot (9 $\mathrm{m} \mathrm{x}$ $5 \mathrm{~m}$ ) with fruit trees (acerola, lemon, mandarin, palms) growing naturally (Valencia, Venezuela). Two fruits during growth from the bottom crown $(2.5 \mathrm{~m}$ high from the ground) of the tree were selected (equatorial diameter $39 \mathrm{~mm}$ ), and a fixed reference for measurements was placed nearby. A laptop computer and a CCD digital camera, (calibrated), were used, and graphic design software. Assays were carried out during 105 days and every 4 to 5 days the fruits were photographed. The images were downloaded to computer and processed with the software CorelDRAW $\AA$ X3. The measurements of fruits in images were done using a virtual graduated rule created with the software, and with the data, growth curves were made. The behaviour of both fruits during growth was similar with coincidences in measurements (day 58: $44.25 \mathrm{~mm}$; day 84: $50.50 \mathrm{~mm}$ ). The phase 3 started at day 84, approximately, and the equatorial diameters reached at day 105 were for each fruit 48.50 y $50.75 \mathrm{~mm}$. Measurements were feasible by the procedure used.

Key words: Digital processing of images, equatorial diameter, fruit growth curves, mandarin. 


\section{INTRODUCCIÓN}

$\mathrm{E}$ crecimiento de los frutos constituye un aspecto de gran importancia en el estudio de la ecofisiología de los cultivos (Freyre et al., 2007). La medida del cambio de diámetro en un fruto es de gran interés en la investigación fisiológica de un árbol integral o de un solo fruto, ya que puede proporcionar información útil para el manejo del huerto o cultivo, como por ejemplo, la dinámica de crecimiento en ciclos bajo distintas condiciones climáticas y/o para estimar el peso a cosecha (Casierra-Posada y Cardozo, 2009). Lo anterior se ve obstaculizado por la dificultad técnica de medición de los cambios de diámetro de los frutos con precisión y al costo de los medidores automáticos (Morandi et al., 2007).

El seguimiento continuo y preciso de los cambios de diámetro de tallos y frutos se ha realizado utilizando medidores automáticos comerciales o construidos por los autores de los estudios específicos llevados a cabo, por ejemplo dendrómetros y/o sensores de diámetro para frutos (Schütte y Burger, 1981; Link et al., 1998; Freyre et al., 2007; Morandi et al., 2007; Biondi y Hartsough, 2010), cuya implementación podría dificultar su uso tanto para investigación como para propósitos prácticos. Asimismo, también se usan calibradores tipo vernier analógicos o digitales (Laguado et al., 2002; LaskowskiOchoa, 2006; Fallas et al., 2010), siempre que la altura de los frutos en la planta lo permita, y en caso contrario, mediante recolección de frutos en diversas etapas de maduración, donde por lo general quedan excluidos los ubicados en el dosel arbóreo, debido a la distancia del suelo; destacando en otro sentido que, para la observación de fenómenos de fructificación se suelen utilizar binoculares y/o telescopios (Parrado-Rosselli et al., 2006; Vílchez et al., 2008).

Una cámara digital con zum variable y software desarrollado de utilidad en el diseño gráfico, permiten obtener información para conocer el modo de crecimiento de los frutos con respecto al tiempo (Padrón-Pereira, 2010). Como hipótesis se plantea que el uso de imágenes digitales y software con capacidad de medición, permite elaborar curvas de crecimiento para el estudio de patrones en frutos de mandarina. Por lo expresado, y tomando en cuenta que las curvas de crecimiento de diámetro ecuatorial versus tiempo son las más empleadas (Avanza et al., 2004), el objetivo de este trabajo fue utilizar imágenes digitales para medir el diámetro ecuatorial en frutos de mandarina durante el crecimiento con la finalidad de aportar un procedimiento alternativo para el estudio de patrones de crecimiento.
Materiales Y MÉTOdos

\section{Área de estudio}

Este trabajo se realizó en una parcela $(9 \mathrm{~m}$ x $5 \mathrm{~m}$ ) plantada con árboles frutales (Acerola, limón, mandarina y palmeras) creciendo naturalmente, en secano y elongados por causa de la competencia interespecífica por la luz, ubicada en el Sector La Pastora, Ciudad de Valencia del Estado Carabobo, Venezuela, coordenadas geográficas $10^{\circ} 10^{\prime} 58^{\prime \prime}$ latitud Norte, $68^{\circ} 0$ '38" longitud Oeste. La fórmula climática de la localidad es CA,PL(U2a, Al), que indica un clima cálido $\left(23\right.$ a $\left.25.9^{\circ} \mathrm{C}\right)$ y poco lluvioso (precipitaciones de 700 a $1199 \mathrm{~mm}$ año $^{-1}$ ), cuyo patrón pluviométrico refiere a un régimen de estación lluviosa uniforme, biestacional y con una variabilidad alta de su precipitación mensual, presentando una estación seca o semiseca y otra lluviosa o semilluviosa, con mayor precipitación desde abril, mayo o junio durante cuatro ó más meses (Silva-León, 2010). El clima antes y durante el tiempo de estudio se caracterizó por lluvias incipientes y constantes. El suelo pertenece al orden inceptisol (Comerma, 1971) o cambisol (FAO et al., 2012).

\section{Equipos y software}

Se usó una computadora portátil (laptop), marca ACER, modelo Aspire 3661WLMi. La captura de imágenes se realizó con una cámara digital, previamente calibrada que aportó un filtro cálido (Padrón-Pereira, 2010), marca Panasonic (LUMIX), modelo DMCFS42; que posee sensor CCD de tamaño 1/2.5 pulgadas, aporta tamaños de imágenes de hasta 10.1 megapíxeles efectivos, dispone de enfoque en modo normal $(50.0 \mathrm{~cm}$ $-\infty)$ y zum óptico variable.

El software utilizado fue CorelDRAW® X3 (Corel Corporation, Ottawa, Ontario, Canada), (que permite medir en unidades de $\mathrm{mm}, \mathrm{cm}, \mathrm{m}$ y $\mathrm{km}$, entre otras) con el que se creó una regla graduada virtual (RGV) de $30 \mathrm{~mm}$ de longitud x $0.5 \mathrm{~mm}$ de ancho con divisiones de $0.25 \mathrm{~mm}$ siguiendo el procedimiento descrito por Padrón-Pereira (2010). La RGV se utilizó para medir el diámetro ecuatorial de los frutos de mandarina en las imágenes captadas y ocasionalmente se comprobó que la medida fuese igual a la de los frutos en el árbol para validar el procedimiento en estos frutos.

\section{Selección de los frutos y referencia fija de medida (RFM)}

En un árbol de mandarina (Citrus reticulata) de altura $6.80 \mathrm{~m}$, con el propósito de dar mayor validez al procedimiento, se seleccionaron 2 frutos de la parte inferior de la copa (Figura 1A). Los criterios de selección fueron: color verde homogéneo (inmaduros), sin rastro de daño físico, en crecimiento y de idéntico diámetro 

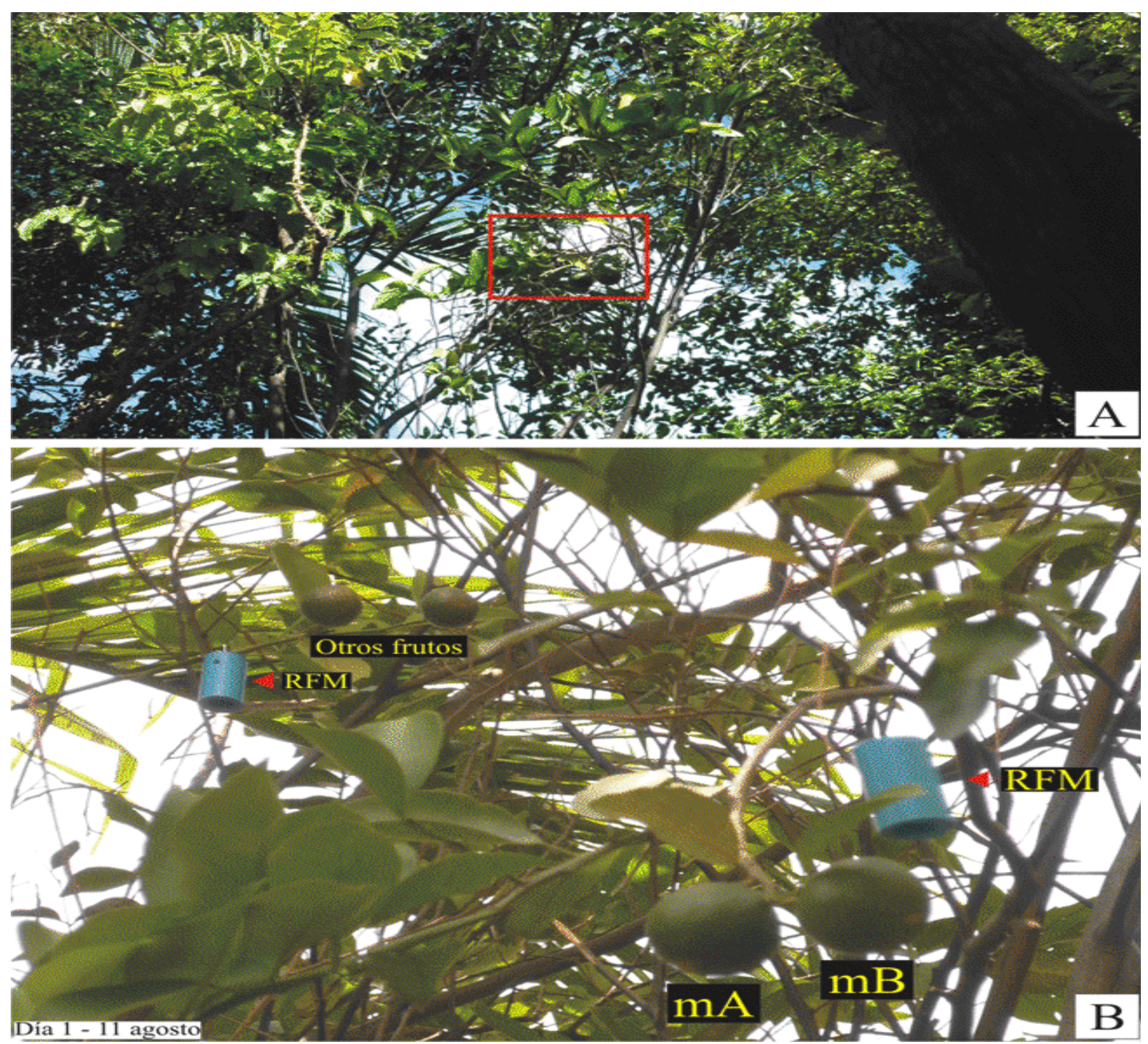

RFM: referencia fija de medida. mA: mandarina A. mB: mandarina B.

Figura 1. Árbol de mandarina con la región de interés enmarcada (A) y ubicación de las RFM en cercanía a los frutos (B)

ecuatorial $(39 \mathrm{~mm})$. Cerca de los frutos se colocó un trozo de tubo plástico azul con diámetro externo de 21.5 $\mathrm{mm}$; que sirvió como RFM. Los frutos se identificaron como mandarina A $(\mathrm{mA})$ y mandarina $\mathrm{B}(\mathrm{mB})$ (Figura 1B).

\section{Medición de los frutos en el tiempo}

Desde el 11 de agosto hasta el 23 de noviembre de 2012 (105 días) en época de lluvias, se tomaron fotografías (zum 5.7X) a los 2 frutos desde un punto fijo de observación a distancia de $1.6 \mathrm{~m}$. Las imágenes presentaron tamaño de 4.9152 megapíxeles $\approx 5$ megapíxeles (2560 x 1920 píxeles) y resolución 180 ppp. Se descargaron en la computadora y se importaron a CorelDRAW ${ }^{\circ}$ X3. Por medio de los nodos de las esquinas de cada imagen, se redujo el tamaño hasta que la medida con la RGV del diámetro del trozo de tubo utilizado como RFM fue igual a $21.5 \mathrm{~mm}$ y seguidamente se midió el diámetro ecuatorial de cada fruto. Para otros frutos que se observan más distantes en una misma imagen, se procede de la misma manera (igualación).

Los 2 frutos se fotografiaron en promedios cada 4 ó 5 días durante el resto de la etapa de crecimiento, hasta la caída de los mismos. Las curvas de crecimiento de mA y $\mathrm{mB}$ se construyeron con los datos obtenidos, siguiendo los cambios de la variable física dependiente (diámetro ecuatorial) en función de la variable independiente (tiempo de evaluación). 


\section{Factibilidad de las mediciones}

La figura 2 ilustra la medición del diámetro ecuatorial de los frutos de mandarina mediante el software CorelDRAW® X3. Se presentan 2 imágenesdetalle captadas con zum (5.7X) donde se aprecia la medición con la RGV, del trozo de tubo utilizado como RFM y del diámetro ecuatorial de los frutos (Figuras 2A y 2B) y una imagen con zum, aumentada en el software, los frutos presentaron igual diámetro ecuatorial (39.0 $\mathrm{mm}$ ) y se comportaron de similar manera durante el crecimiento, con coincidencias en las mediciones a los
58 y 84 días (44.25 y $50.50 \mathrm{~mm}$, respectivamente). La abscisión ocurrió a los 106 días para mA y 110 días para $\mathrm{mB}$. En frutos, las tasas de crecimiento y desarrollo están determinadas por condiciones climáticas, la variedad cultivada (Orduz-Rodríguez et al., 2009) y prácticas de manejo precosecha (Din et al., 2012).

El patrón de crecimiento de los frutos de mandarina describe una curva sigmoidea (Wongchana y Pongmanawut, 2003; Ladaniya, 2008) como otros frutos del género Citrus, dividida en tres fases sucesivas, siendo difícil delimitar con exactitud el final de una e

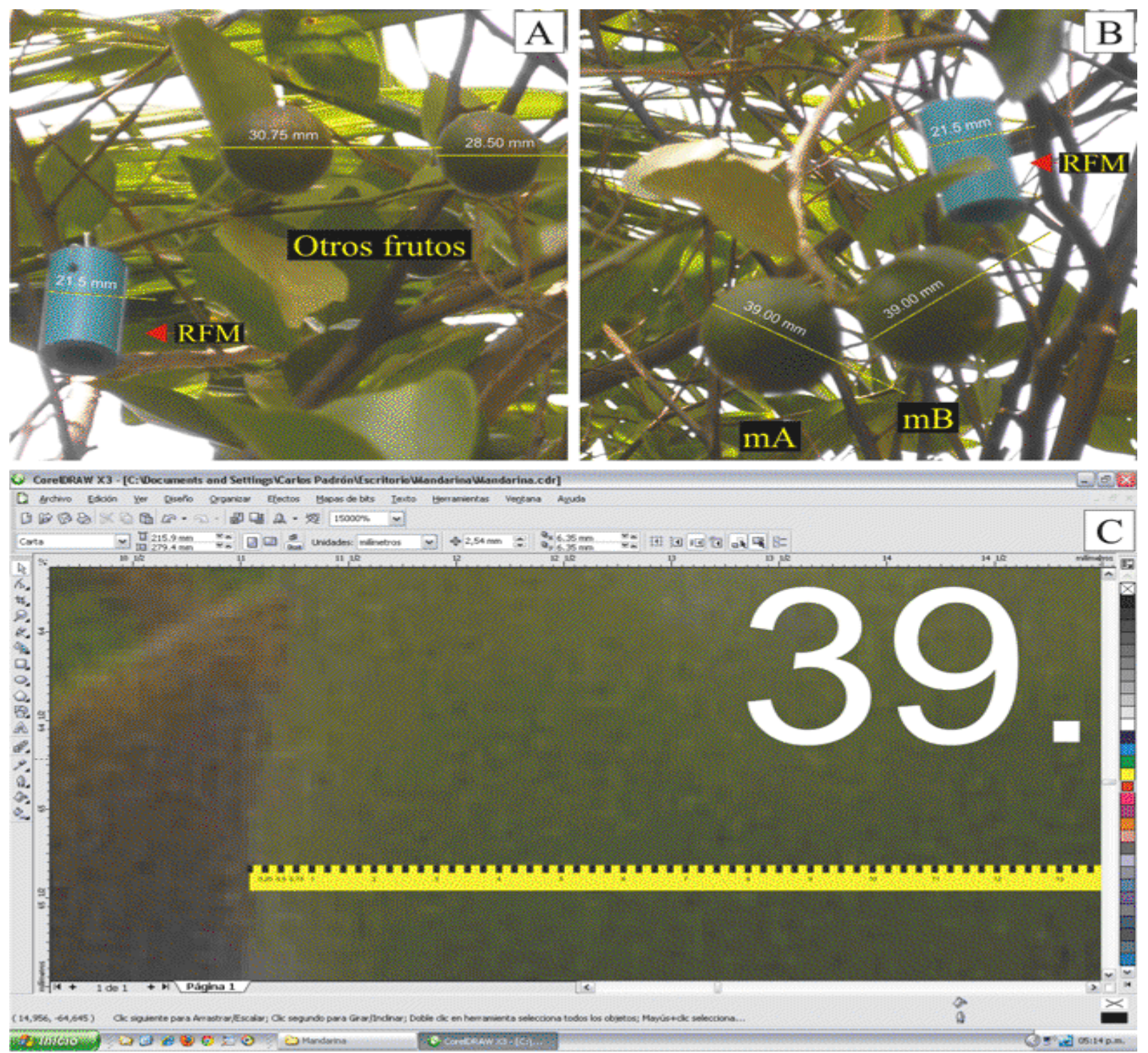

RFM: referencia fija de medida. $\mathrm{mA}$ : mandarina A. mB: mandarina B.

Figura 2. Medición del diámetro ecuatorial en frutos de mandarina. Medición de frutos inicialmente distantes en la imagen, igualados (A). Medición de los frutos estudiados, el primer día (B). Imagen (zum 5.7X) aumentada en el software al $15000 \%(C)$ 
Cuadro 1. Valores medidos en la evolución del crecimiento en el árbol de los 2 frutos de mandarina evaluados

\begin{tabular}{rrcc}
\hline Día & Fecha & $\mathbf{m A}(\mathbf{m m})$ & $\mathbf{m B}(\mathbf{m m})$ \\
\hline 1 & 11-Aug & 39.00 & 39.00 \\
5 & 15-Aug & 39.25 & 39.75 \\
9 & 19-Aug & 39.00 & 40.25 \\
14 & 24-Aug & 39.50 & 42.75 \\
18 & 28-Aug & 40.50 & 41.75 \\
21 & 31-Aug & 41.25 & 42.50 \\
26 & 5-Sep & 41.50 & 42.25 \\
30 & 9-Sep & 41.25 & 43.25 \\
36 & 15-Sep & 41.75 & 43.00 \\
39 & 18-Sep & 42.50 & 43.00 \\
48 & 27-Sep & 41.75 & 42.75 \\
53 & 2-Oct & 43.50 & 44.50 \\
58 & 7-Oct & 44.25 & 44.25 \\
62 & 11-Oct & 43.00 & 44.25 \\
66 & 15-Oct & 45.25 & 46.25 \\
71 & 20-Oct & 46.50 & 47.75 \\
78 & 27-Oct & 49.25 & 49.75 \\
84 & 2-Nov & 50.50 & 50.50 \\
91 & 9-Nov & 50.50 & 49.75 \\
94 & 12-Nov & 49.50 & 50.50 \\
100 & 18-Nov & 48.25 & 49.00 \\
105 & 23-Nov & 48.50 & 50.75 \\
106 & 24-Nov & abscisión & \\
110 & 28-Nov & & abscisión \\
\hline & & \\
& &
\end{tabular}

$\mathrm{mA}$ : mandarina A. mB: mandarina B.

inicio de la siguiente (Esposti et al., 2008). La fase 1, un intervalo de aproximadamente dos meses de división celular y lento crecimiento desde la antesis; la fase $2 \mathrm{o}$ de crecimiento rápido, en donde el fruto experimenta un gran incremento en el tamaño por expansión celular y acumulación de agua durante cuatro a seis meses, y, la fase 3 o período de maduración, donde el crecimiento en su mayoría se detiene (Dalal et al., 2013).

El intervalo de la fase 2 y la fase 3 , con participación en el sigmoideo, se delimitan en la figura 3 . La fase 3 inició aproximadamente el día 84 de manera coincidente en ambos frutos y la fase 1 no fue estudiada, donde las tendencias de las curvas se asemejan a las curvas de crecimiento de frutos de Citrus reticulata Blanco graficada por Esposti et al. (2008) a partir de 85 ddpf (días después de plena floración) y de frutos de Citrus sinensis var. Valencia, representadas por Avanza et al. (2004) a partir de 66 ddpf (autores que no estudiaron la fase 1).

Los diámetros ecuatoriales alcanzados a los 105 días fueron $48.50 \mathrm{~mm}$ en $\mathrm{mA}$ y $50.75 \mathrm{~mm}$ en $\mathrm{mB}$ (Cuadro 1; figuras 3 y 4). El fruto $\mathrm{mA}$ aunque de menor diámetro, fue el que presentó mejor desarrollo de color (Figura 4) y abscisión más temprana (Cuadro 1). Frutos de determinado diámetro pueden presentar diversos colores de estados de maduración (Padrón-Pereira, 2010).

Para otros frutos del mismo árbol estudiado, el día previo a la abscisión midieron 62.50 y $78.25 \mathrm{~mm}$. Para un mismo cultivar de mandarina, Din et al. (2012) determinaron diámetros ecuatoriales de 64.33 a 71.58 mm; Dalal et al. (2013) 71.34 mm; y Altaf y Khan (2009) $78.50 \mathrm{~mm}$. Un mismo cultivar de mandarina puede presentar extrema variabilidad en todas las características del fruto (Altaf et al., 2008; Din et al., 2012). Se ha reportado que en cítricos (Citrus sinensis L. Osbeck), las condiciones limitadas de riego son responsables de un número mayor de frutos pequeños (menor diámetro final) (Treeby et al., 2007), aunque por el contrario, en árboles de mandarina 'Fortune' se ha observado que el tamaño del fruto no se vio afectado por la restricción de agua (Dell'Amico-Rodríguez et al., 2012). En presencia de lluvias incipientes y constantes, antes y durante el período estudiado, se produjeron frutos pequeños, medianos y grandes.

Entre las posibles causas de las respuestas observadas, el crecimiento de los frutos cítricos está determinado por factores endógenos (Genéticos, posición del fruto, competencia entre órganos en desarrollo) y exógenos (Ambientales y prácticas culturales). En un mismo árbol, hojas jóvenes cercanas al brote actúan como órganos competidores de los frutos, mientras que hojas maduras adquieren un papel esencial como fuente de fotosintatos; lo que estimula el desarrollo del fruto (Moss et al., 1972; SpiegelRoy y Goldschmidt, 1996; Agustí et al., 2003). Para el día 1 los frutos presentaban igual diámetro y en la cercanía, $\mathrm{mA}$ una hoja madura y $\mathrm{mB}$ una hoja joven (Figura 2B). El día 14 ocurrió la caída natural de la hoja en $\mathrm{mA}$ mientras que en $\mathrm{mB}$ la hoja se mantuvo hasta el día 105, como también es de hacer notar que este fruto fue el que creció en el extremo terminal de la rama (Figura 4). En razón de lo anterior, es probable que la presencia inicial de la hoja madura en $\mathrm{mA}$, la caída de la hoja (menor competencia) y la posición del fruto en un extremo menos apical, fueron causas que contribuyeron con el mayor desarrollo del fruto, independientemente de haber presentado un menor diámetro ecuatorial. Con respecto a $\mathrm{mB}$, desde el principio fue el fruto que 
Padrón-Pereira

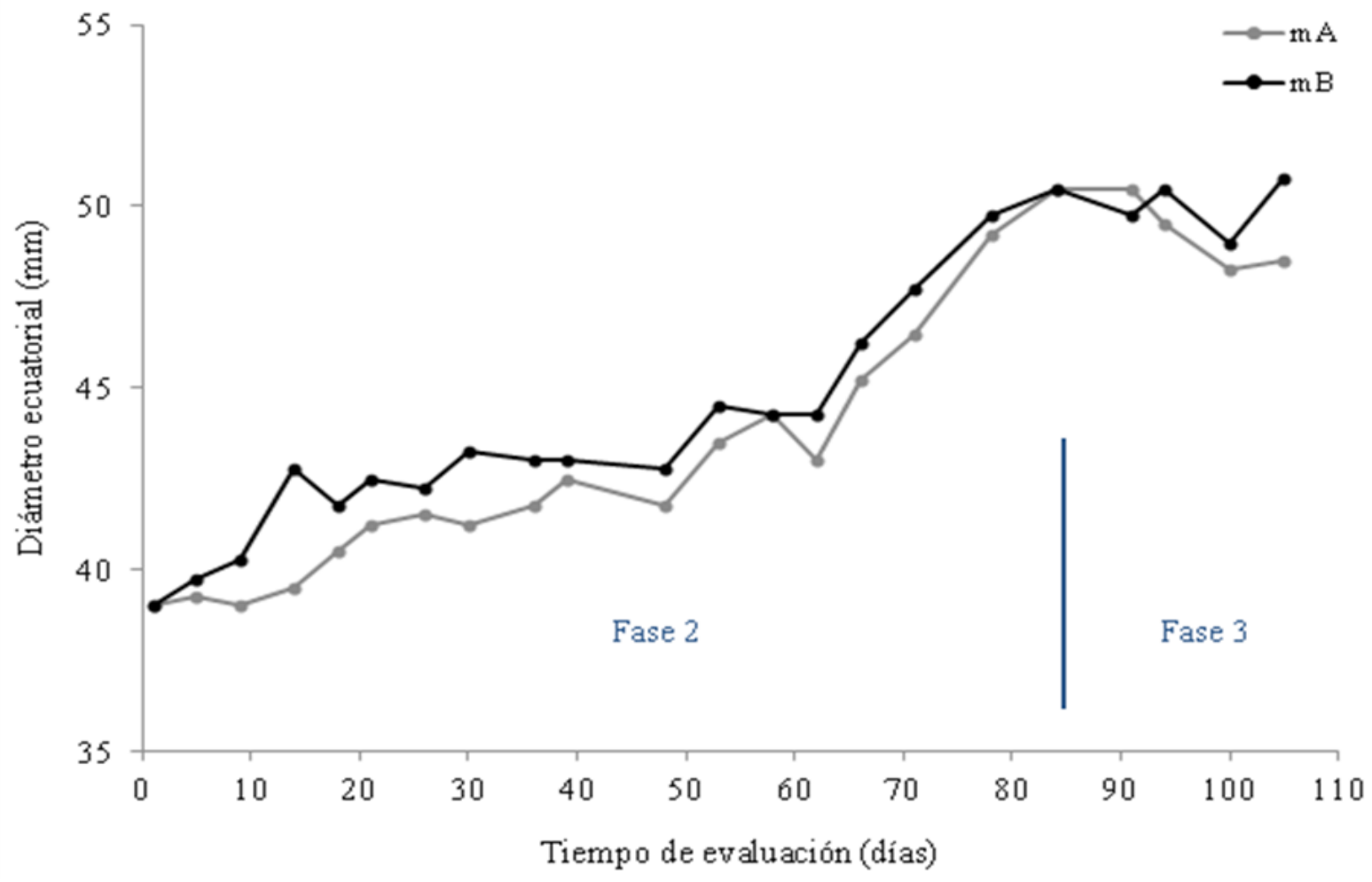

Figura 3. Curvas de crecimiento en el árbol de los 2 frutos de mandarina

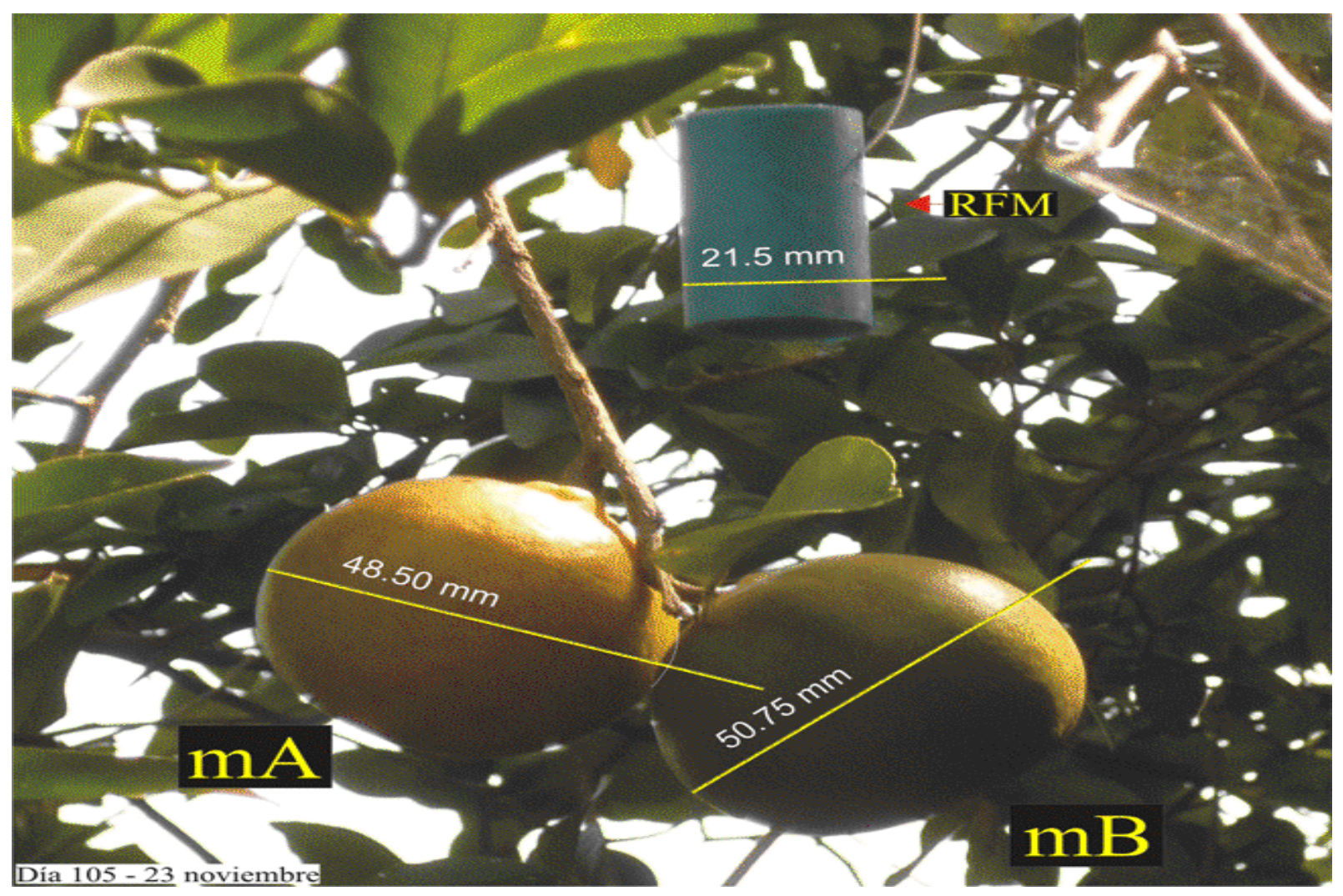

RFM: referencia fija de medida. $\mathrm{mA}$ : mandarina A. $\mathrm{mB}$ : mandarina B.

Figura 4. Medición de los frutos estudiados, el último día 
presentó mayores valores de diámetro ecuatorial durante el tiempo de evaluación (Figura 3). Tendencia hacia un mayor crecimiento con retardo en la maduración permite al fruto crecer por más tiempo (Duarte-B., 1980). Esta regulación del desarrollo depende de la actividad hormonal; biosíntesis por el propio fruto de diversas hormonas, como del transporte a él o hacia otras partes de la planta promoviendo procesos en base a la expresión de la información genética (Agustí, 2010).

Medina-Urritia et al. (2004) describen que en cítricos, a mayor densidad de plantación, el rendimiento en producción de frutos por árbol se reduce por la competencia intraespecífica, pero el tamaño de los frutos no es afectado, y en algunas ocasiones se obtienen frutos un poco más pequeños en altas densidades.

En otro sentido, el procesamiento digital de imágenes ha permitido la medición del área de plantas en crecimiento (Green et al., 2012) y la geometría de órganos de plantas, como las raíces (French et al., 2012). Padrón-Pereira (2010), empleando el mismo procedimiento utilizado en este trabajo, elaboró curvas de crecimiento de diámetro ecuatorial en función del tiempo para frutos de semeruco (Malpighia glabra L.), los cuales se caracterizan por su rápido desarrollo y maduración.

Todo lo expuesto evidencia la factibilidad del procedimiento empleado, para la realización de mediciones del diámetro ecuatorial en frutos de mandarina (De desarrollo más lento), lo que a su vez permitió representar y describir la evolución del crecimiento de los frutos en el árbol. El procedimiento reviste interés como aplicación práctica para la observación fitofenológica, en casos donde se dificulte la misma cuando el propósito es medir. Además de la medición de diámetros en frutos ubicados en la periferia o el interior de la copa de un árbol que se encuentren a considerable altura del suelo, reviste interés para la adquisición de datos con miras a la aplicación de modelos matemáticos para análisis del crecimiento de frutos de mandarina y otros sin muestreos destructivos, en la medición de la altura y diámetro de copa de árboles entre otros parámetros morfométricos y en la fotogrametría de cultivos.

\section{Conclusiones}

L os dos frutos de mandarina evaluados, en las fases de $u_{\text {crecimiento, presentaron comportamiento similar }}$ en parte al sigmoideo. El procedimiento empleado permitió la medición del diámetro ecuatorial de frutos de mandarina utilizando imágenes digitales y se presenta como aplicación práctica para la observación fenológica en la fase de fructificación de la mandarina.

\section{Agradecimientos}

El autor agradece a la Asociación RVCTA, el hardware, software y el aporte económico para el desarrollo del trabajo.

\section{Literatura CITADA}

Agustí, M. 2010. Fruticultura (2da. Ed.). Ediciones Mundi-Prensa, Madrid, España.

Agustí, M., A. Martínez-Fuentes, C. Mesejo, J. Mariano y V. Almela. 2003. Cuajado y Desarrollo de los Frutos Cítricos. Sèrie Divulgació Tècnica No 55 . Consellería de Agricultura, Pesca y Alimentación, Generalitat Valenciana, España.

Altaf, N., and A. R. Khan. 2009. Growth and development of low seeded Kinnow mandarin fruits in dense plantation. Journal of Agricultural Science and Technology. 11(2):191-198.

Altaf, N., A. R. Khan, and J. Hussain. 2008. Fruit variability in Kinnow mandarin (Citrus reticulata). Pakistan Journal of Botany. 40(2):599-604.

Avanza, M. M., L. Giménez, S. M. Mazza y V. A. Rodríguez. 2004. Descripción del crecimiento de frutos de naranjo dulce mediante el uso de modelos no lineales. Comunicaciones Científicas y Tecnológicas, Resumen: A-018. Universidad Nacional del Nordeste, Argentina.

Biondi, F., and P. Hartsough. 2010. Using automated point dendrometers to analyze tropical treeline stem growth at Nevado de Colima, Mexico. Sensors. 10(6):5827-5844.

Casierra-Posada, F. y M. C. Cardozo. 2009. Análisis básico del crecimiento en frutos de tomate (Lycopersicon esculentum Mill, cv. "Quindío") cultivados a campo abierto. Revista Facultad Nacional de Agronomía, Medellín. 62(1):48154822.

Comerma, J. A. 1971. La 7a aproximación y los suelos venezolanos. Agronomía Tropical. 21(5):365-377.

Dalal, R. P. S., B. S. Beniwal and S. K. Sehrawat. 2013. Seasonal variation in growth, leaf physiology and fruit development in Kinnow, a mandarin hybrid. Journal of Plant Studies. 2(1):72-77.

Dell'Amico-Rodríguez, J. M., R. Domingo-Miguel, A. Pérez-Pastor, M. García, M. Peñalver, F. Villanueva y P. Puerto. 2012. Efecto del estrés hídrico em El desarrollo final Del fruto de mandarina 'Fortune'. Cultivos Tropicales. 33(3):63-68.

Din, A., M. Asghar, S. Parveen and M. H. Ali. 2012. Evaluation of Kinnow mandarin as influenced by pre-harvest management practices. Journal of Agricultural Research. 50(3):381-392. 
Duarte-B., O. 1980. Reguladores de Crecimiento en Cítricos. En: Morín-L., C (Ed.). Cultivo de Cítricos (2da. Ed.). Instituto Interamericano de Ciencias Agrícolas de la Organización de Estados Americanos. pp 416.

Esposti, M. D. D., D. L. de Siqueira e P. R. Cecon. 2008. Crescimento de frutos da tangerineira 'Poncã' (Citrus reticulata Blanco). Revista Brasileira de Fruticultura. 30(3):657-661.

Fallas, R., F. Bertsch, E. Miranda y C. Henríquez. 2010. Análisis de crecimiento y absorción de nutrimentos de frutos de mango, cultivares Tommy Atkins y Keith. Agronomía Costarricense. 34(1):1-15.

FAO (Food and Agriculture Organization of the United Nations); ISS/CAS (Institute of Soil Science/Chinese Academy of Sciences); IIASA (International Institute for Applied Systems Analysis); ISRIC (International Soil Reference and Information Centre) and JRC (Joint Research Centre of the European Commission). 2012. Harmonized World Soil Database (HWSD) version 1.21. FAO, Rome, Italy - IIASA, Laxenburg, Austria.

French, A. P., M. H. Wilson, K. Kenobi, D. Dietrich, U. Voß, S. Ubeda-Tomás, T. P. Pridmore and D. M. Wells. 2012. Identifying biological landmarks using a novel cell measuring image analysis tool: cell-o-tape. Plant Methods. 8:7(2 March). 7 p.

Freyre, C. E., P. A. Schapschuk, C. A. Bouzo y E. R. Bouchet. 2007. Construcción de un dendómetro y una estación meteorológica automática para el estudio del crecimiento de frutos. Revista Científica Agropecuaria. 11(2):103-109.

Green, J. M., H. Appel, E. M. Rehrig, J. Harnsomburana, J. F. Chang, P. Balint-Kurti and C. R. Shyu. 2012. PhenoPhyte: a flexible affordable method toquantify 2D phenotypes from imagery. Plant Methods. 8:45(6 November). 12 p.

Ladaniya, M. 2008. Citrus Fruit: Biology, Technology, and Evaluation. Academic Press, San Diego, CA, USA.

Laguado, N., M. Marín, L. Arenas de Moreno, F. Araujo, C. Castro de Rincón y A. Rincón. 2002. Crecimiento del fruto de guayaba (Psidium guajava L.) del tipo Criolla Roja. Revista de la Facultad de Agronomía (LUZ). 19(4):273-283.

Laskowski-Ochoa, L. E. 2006. Características de la abscisión del fruto de naranja Citrus sinensis (L.) Osbeck cv. Salustiana. Bioagro. 18(1):25-30.

Link, S. O., M. E. Thiede and M. G. van Bavel. 1998. An improved strain-gauge device for continuous field measurement of stem and fruit diameter. Journal of Experimental Botany. 49(326):1583-1587.
Medina-Urritia, V. M., S. Becerra-Rodríguez y E. Ordaz-Ordaz. 2004. Crecimiento y rendimiento del limón mexicano en altas densidades de plantación en el trópico. Revista Chapingo serie horticultura. 10(1):43-49.

Morandi, B., L. Manfrini, M. Zibordi, M. Noferini, G. Fiori and L. Corelli-Grappadelli. 2007. A low-cost device for accurate and continuous measurements of fruit diameter. HortScience. 42(6):1380-1382.

Moss, G.I., B. T. Steer, and P. E. Kriedemann. 1972. The regulatory role of inflorescence leaves in fruit-setting by sweet orange (Citrus sinensis). Physiologia Plantarum. 27(3):432-438.

Orduz-Rodríguez, J. O., H. Monroy, G. Fischer y A. Herrera-A. 2009. Crecimiento y desarrollo del fruto de mandarina (Citrus reticulata) 'Arrayana' en condiciones del piedemonte del Meta, Colombia. Revista Colombiana de Ciencias Hortícolas. 3(2):149-160.

Padrón-Pereira, C. A. 2010. Procesamiento digital de imágenes de frutos de semeruco (Malpighia glabra L.) durante el crecimiento y maduración. Revista Científica Eletrónica de Agronomía. 17(2):1-17.

Parrado-Rosselli, Á., J. L. Machado and T. PrietoLópez. 2006. Comparison between two methods for measuring fruit production in a tropical forest. Biotropica. 38(2):267-271.

Schütte, K. H., and C. P. Burger. 1981. Sensitive dendrometers for contemporary research. A critical evaluation of strain gauge dendrometers. Journal of South African Botany. 47:273-291.

Silva-León, G. A. 2010. Tipos y subtipos climáticos de Venezuela. Trabajo de ascenso a la categoría de titular. Escuela de Geografía, Facultad de Ciencias Forestales y Ambientales, Universidad de Los Andes. Mérida, República Bolivariana de Venezuela.

Spiegel-Roy, P., and E. E. Goldschmidt. 1996. Biology of Citrus. Cambridge University Press, New York, USA.

Treeby, M. T., R. E. Henriod, K. B. Bevington, D. J. Milne and R. Storey. 2007. Irrigation management and rootstocks effects on navel orange [Citrus sinensis (L.) Osbeck] fruit quality. Agricultural Water Management. 91(1-3):24-32.

Vílchez, B., R. Chazdon y W. Alvarado. 2008. Fenología reproductiva de las especies del dosel en bosques secundarios y primarios de la región Huetar Norte de Costa Rica y su influencia en la regeneración vegetal. Kurú: Revista Forestal. 5(15):18 p.

Wongchana, B. and D. Pongmanawut. 2003. Studies on growth and development of neck orange fruit (Citrus reticulata Blanco). Thai Agricultural Research Journal. 21(2):97-104. 
Pettinelli Junior e M. Sakai. 1985. Identificação de progênies de arroz resistentes à brusone no Estado de São Paulo, em 1976-1977. Bragantia 44(1):311329.

Webster, R. K. and P. S. Gunnell. 1992. Compendium of Rice Diseases. The American Phytopathological Society-APS, St. Paul. 62 p.

Zambolim, L., W. S. Venâncio, S. H. Furlan de Oliveira. 2007. Manejo de Resistência de Fungos a Fungicidas. Suprema Gráfica e Editora, Visconde do Rio Branco. 168 p. 